\title{
Ajuste e avaliação na estimativa volumétrica para Lecythis lurida (Miers) S.A. Mori em uma área de manejo florestal
}

\author{
Girlene da Silva Cruz ${ }^{*}$ Renato Bezerra da Silva Ribeiro ${ }^{2}$ João Ricardo Vasconcellos Gama ${ }^{2}$ Bruno Rafael Silva \\ de Almeida ${ }^{1}$ Lucas Cunha Ximenes ${ }^{2}$ Karla Mayara Almada Gomes ${ }^{3}$ Talita Godinho Bezerra ${ }^{3}$
}

\begin{abstract}
${ }^{1}$ Programa de Pós-Graduação em Engenharia Florestal (PPGEF), Universidade do Estado de Santa Catarina, Centro de Ciências Agroveterinárias, Av. Luiz de Camões, 2090 - Conta Dinheiro, Lages - SC, 88520-000

2 Programa de Pós-Graduação em Sociedade, Natureza e Desenvolvimento, Universidade Federal do Oeste do Pará, Rua Vera Paz, s/n, Salé, Santarém-PA, 68040-255 ${ }^{3}$ Programa de Pós-Graduação em Ciências Florestais, Instituto de Ciências Agrárias, Universidade Federal Rural da Amazônia, Av Presidente Tancredo Neves, 2501,
\end{abstract} Terra Firme, Belém-PA, 66.077-830

*Author for correspondence: scruz.girlene@gmail.com

Received: September 2018 / Accepted: February 2019 / Published: March 2019

\section{Resumo}

O objetivo do presente estudo foi avaliar a eficiência de ajustes volumétricos para Lecythis lurida na Floresta Nacional do Tapajós. Os dados foram provenientes de inventário $100 \%$ e cubagem rigorosa de espécies na UPA 8. Foram selecionadas 301 árvores amostras de Lecythis lurida com $50 \mathrm{~cm} \geq$ DAP $<150 \mathrm{~cm}$, no qual foram ajustados 8 modelos volumétricos. Para a escolha da melhor equação, foram analisados a significância dos coeficientes pelo teste t, o coeficiente de determinação ajustado, o erro padrão da estimativa, o fator de inflação da variância para verificar situações de multicolinearidade, a análise gráfica e histogramas de resíduos. A padronização dos resíduos foi realizada para as melhores equações. Para a validação da melhor equação, o volume real e o estimado foram comparados pelo teste Qui quadrado a 95\% de probabilidade. Os modelos Spurr variável combinada, Husch, Schumacher Hall e Spurr - ambos logaritmizados - apresentaram significância de todos os parâmetros pelo teste t. Em relação aos parâmetros de precisão os modelos de Spurr variável combinada, Schumacher Hall e Spurr logaritmizados apresentaram os melhores valores. Após a retirada de outliers é nítida a melhora nos parâmetros de precisão dos modelos. Considerando todos os critérios de seleção, o modelo de Spurr variável combinada pode ser selecionado para estimar a volumetria da espécie na área de estudo, além disto, a equação selecionada gerou estimativas que não diferiram estatisticamente com volume real segundo o teste qui quadrado.

Palavras-chave: Análise de regressão, Jarana, Manejo Florestal Comunitário.

\begin{abstract}
The objective of the present study was to evaluate the efficiency of volumetric adjustments for Lecythis lurida in the Tapajos National Forest. The data came from $100 \%$ inventory and and rigorous species counting in UPA 8. 301 trees were selected from Lecythis lurida with $50 \mathrm{~cm} \geq$ DAP $<150 \mathrm{~cm}$, where eight volumetric models were fitted. For the choice of the best equation, we analyzed the significance of the coefficients by the $t$ test, adjusted coefficient of determination, standard error of the estimate, and factor of inflation of the variance to verify situations of multicollinearity, graphical analysis and histograms of residues. For the validation of the best equation, the actual and estimated volume were compared by the chi-square test at $95 \%$ probability. The combined Spurr variable models, Husch, Schumacher Hall and Spurr - both logarithmized presented significance of all parameters by the $t$ test. Regarding the precision parameters, the Spurr combined variable models, Schumacher Hall and Spurr logarithmized presented the best values. After the removal of outliers is,
\end{abstract}

clear the improvement in the parameters of precision of the models. After the removal of outliers is clear the improvement in the parameters of precision of the models. Considering all the selection criteria, the combined variable Spurr model can be selected to estimate the volumetry of the species in the study area. The selected equation generated estimates that did not differ statistically with real volume according to the chi-square test.

Keywords: Regression analysis, Jarana, Community Forest Management.

\section{Introdução}

$\mathrm{O}$ ajuste de equações de volume constitui-se como o procedimento mais adotado e eficiente para a quantificação da produção em um maciço florestal (Guimarães e Leite 1996; Silva et al. 2009; Cabacinha et al. 2013). Para sua realização, algumas árvores são abatidas e, em seguida, passam por um processo conhecido como cubagem rigorosa, para, posteriormente, ajustar as equações de regressão e estimar a produção volumétrica da floresta (Couto e Bastos 1987; Azevedo et al. 2011; Cabacinha et al. 2013).

Diversos modelos foram desenvolvidos e aplicados em diferentes regiões do Brasil para esse fim, porém, é recomendável que sempre sejam testados vários modelos matemáticos e, por meio de análise estatística, escolhido o melhor a ser aplicado. Recomenda-se também utilizar equações específicas para cada local, pois nem sempre modelos já "consagrados" se ajustam a todas as espécies e ambientes (Thomas et al. 2006; Barreto et al. 2014).

$\mathrm{O}$ desenvolvimento e o aprimoramento de equações de volume para a Amazônia são importantes para subsidiar as ações de planejamento florestal. As áreas em concessão requerem estimativas precisas do volume comercial das árvores, desde a avaliação da floresta para o embasamento das licitações, até a emissão da autorização de exploração por parte dos órgãos ambientais competentes, justificando assim o aprimoramento dessas equações e fazendo com que os volumes estimados sejam mais próximos dos volumes retirados das áreas de colheita florestal (Cysneiros et al. 2017).

De acordo com a legislação vigente, no primeiro ano de exploração de uma área florestal não é obrigatória a apresentação de uma equação volumétrica específica para a área a ser manejada, sendo comum a estimativa do volume utilizando o fator de forma 0,7 (Semas 2015). Porém, de acordo com a resolução CONAMA n n $^{\circ}$ 406/2009, a partir do segundo plano de operação anual (POA) é obrigatório que seja ajustada e apresentada uma equação específica para a área manejada, possibilitando assim maior controle sobre a produção das florestas nativas na Amazônia (Brasil 2009). Além disso, minimiza a possibilidade do comércio ilegal de 
créditos de volume de madeira, fato ainda existente na região amazônica (Silva-Ribeiro et al. 2014).

A Floresta Nacional do Tapajós (FNT) é uma Unidade de Conservação Federal, enquadrada na categoria de Uso Sustentável (Brasil 2000), que detém a maior referência de manejo florestal comunitário do Brasil (Oliveira et al. 2017). Desde do ano de 2009, a Cooperativa passou a estimar a volumetria da área de manejo através de equações de volume, vale ressaltar que a cooperativa utiliza uma única equação de dupla entrada para estimar a volumetria de todas as espécies a serem exploradas na área de manejo (Gomes et al. 2018).

Dentre as espécies que são colhidas pela COOMFLONA, Lecythis lurida Miers S.A. Mori., está entre as dez mais importantes e de maior volumetria comercializada na região (Andrade et al. 2015). Lecythis lurida é uma espécie pertencente à família Lecythidaceae conhecida popularmente como Jarana. Frequentemente encontrada em florestas naturais na Amazônia, possui madeira muito pesada, de cor marrom escuro, textura fina a média (Trevisor 2011; Taffarel et al. 2014)

Levando isso em consideração e atrelado ao fato de que equações volumétricas ajustadas por espécie de forma individual ou para um grupo de espécies com características similares garantem maior precisão nas estimativas volumétricas de um maciço florestal (Soares et al. 2011), o objetivo do presente estudo foi avaliar a eficiência de ajustes volumétricos para Lecythis lurida em uma área de manejo florestal na Floresta Nacional do Tapajós.

\section{Material e métodos}

\section{Área de estudo}

Os dados foram provenientes do inventário $100 \%$ e do romaneio de toras realizados na Unidade de Produção Anual 8 (UPA 08), da Área de Manejo Florestal "Samambaia" da Cooperativa Mista da Flona Tapajós (COOMFLONA), que possui concessão não onerosa e pratica Manejo Florestal Comunitário na Floresta Nacional do Tapajós sob uma intensidade de corte de $30 \mathrm{~m}^{3}$.ha-1 (Figura 1).

De acordo com a classificação de Köppen, a FNT localiza-se em região de clima Am, com temperatura anual de $25,5^{\circ} \mathrm{C}$ e umidade relativa do ar de $90 \%$ (Ibama 2004). Em média, $1820 \mathrm{~mm}$ de chuva são esperados por ano, sendo os meses de janeiro a maio, os de maior precipitação. O solo é em sua maioria do tipo Latossolo Amarelo Distrófico com topografia variando de suavemente ondulada a ondulada (Ibama 2004). Na região, predomina floresta do tipo Ombrófila Densa, caracterizada pela dominância de indivíduos arbóreos de grande porte (Pinho et al. 2009; IBGE 2012).

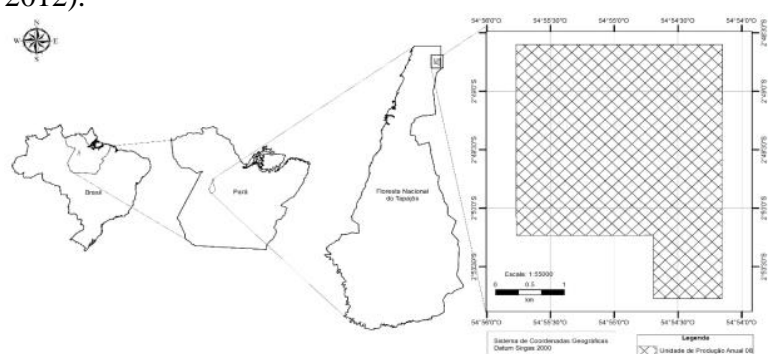

Figura 1. Mapa de localização da Unidade de Produção Anual 8 na Floresta Nacional do Tapajós, Estado do Pará

\section{Coleta e análise de dados}

Foram utilizadas nos ajustes 301 árvores-amostra de Lecythis lurida com $50 \mathrm{~cm} \geq \mathrm{DAP}<150 \mathrm{~cm}$, cubadas pelo método de Smalian e que tiveram o seccionamento de toras variando entre 4 a $7 \mathrm{~m}$ de acordo com metodologia de SilvaRibeiro et al. (2014), onde a soma dos comprimentos das toras, corresponderam a altura comercial de cada árvore. Com os dados observados de volume real, foram ajustados 8 modelos volumétricos para a espécie (Tabela 1 ).

Tabela 1. Modelos volumétricos testados para Lecythis lurida na Área de Manejo Florestal da COOMFLONA, Floresta Nacional do Tapajós.

\begin{tabular}{|c|c|c|}
\hline $\mathrm{N}^{\circ}$ & Autor & Modelo \\
\hline 1 & $\begin{array}{l}\text { Spurr variável } \\
\text { combinada }\end{array}$ & $\mathrm{V}=\beta 0+\beta 1 .\left(\mathrm{D}^{2} . \mathrm{H}\right)+\varepsilon$ \\
\hline 2 & $\begin{array}{l}\text { Spurr } \\
\text { logaritmizado }\end{array}$ & $\ln \mathrm{V}=\beta 0+\beta 1 .\left(\ln ^{2} \cdot \mathrm{H}\right)+\varepsilon$ \\
\hline 3 & Husch & $\ln \mathrm{V}=\beta 0+\beta 1 .(\ln \mathrm{D})+\varepsilon$ \\
\hline 4 & $\begin{array}{l}\text { Hohenald - } \\
\text { Krenn }\end{array}$ & $\mathrm{V}=\beta 0+\beta 1 .(\mathrm{D})+\beta 2 .\left(\mathrm{D}^{2}\right)+\varepsilon$ \\
\hline 5 & $\begin{array}{l}\text { Schumacher Hall } \\
\text { logaritmizado }\end{array}$ & $\ln \mathrm{V}=\beta 0+\beta 1 .(\ln \mathrm{D})+\beta 2 .(\ln \mathrm{H})+\varepsilon$ \\
\hline 6 & Stoate & $\begin{array}{l}V=\beta 0+\beta 1 .\left(D^{2}\right)+\beta 2 .\left(D^{2} \cdot H\right)+\beta 3 .(D \cdot H)+ \\
\beta 4 .(H)+\varepsilon\end{array}$ \\
\hline 7 & $\begin{array}{l}\text { Meyer } \\
\text { modificado }\end{array}$ & $\begin{array}{l}V=\beta 0+\beta 1 .(D)+\beta 2 .\left(D^{2}\right)+\beta 3 .(D \cdot H)+\beta 4 \\
\left(D^{2} \cdot H\right)+\varepsilon\end{array}$ \\
\hline 8 & Meyer & $\begin{array}{l}V=\beta 0+\beta 1 .(D)+\beta 2 .\left(D^{2}\right)+\beta 3 .(D \cdot H)+\beta 4 . \\
\left(D^{2} \cdot H\right)+\beta 5 .(H)+\varepsilon\end{array}$ \\
\hline
\end{tabular}

Em que: $\mathrm{V}=$ volume comercial com casca em $\mathrm{m}^{3} ; \mathrm{D}=$ diâmetro à $1,30 \mathrm{~m}$ do solo, em $\mathrm{cm} ; \mathrm{H}=$ soma do comprimento das toras, em $\mathrm{m}$; $\beta 0, \beta 1, \beta 2, \beta 3, \beta 4, \beta 5=$ coeficientes da regressão; $\ln =$ logaritmo neperiano; $\varepsilon=$ erro aleatório.

Como critérios para a escolha do melhor modelo, foram avaliados a significância dos parâmetros pelo teste $t \mathrm{a} 95 \%$ de probabilidade, análise gráfica dos resíduos, histogramas dos resíduos e os valores percentuais do coeficiente de determinação ajustado ( $\left.\mathrm{R}^{2} \mathrm{aj} \%\right)$ e erro padrão da estimativa (Sy.x\%).

A multicolinearidade foi analisada por meio do VIF (Variance inflation factor) para modelos que apresentam mais de uma variável independente. Geralmente, VIF > 10 significa que o modelo apresenta multicolinearidade problemática, ou seja, a correlação entre as variáveis está afetando os valores dos coeficientes dos modelos.

Para o melhor modelo ajustado foi aplicado o método de padronização dos resíduos que consiste em identificar e retirar os possíveis outliers. Para isso, adotou um valor tabelado de 1,96, conforme metodologia descrita por Scolforo (2005). Após a retirada dos possíveis outliers o melhor modelo foi reajustado. Para a comparação entre as equações logarítmicas e não logarítmicas, o erro padrão e o coeficiente de determinação ajustado foram recalculados. Os modelos volumétricos foram ajustados pelo método dos mínimos quadrados ordinários (MMQO).

O processamento, análise de dados e os testes aplicados foram realizados utilizando planilha eletrônica do Microsoft Excel 2013 e a extensão Action 2.8

\section{Validação da melhor equação}

Realizou-se a validação das equações com 50 árvores amostras, que não fizeram parte dos ajustes. $\mathrm{O}$ volume real e o estimado foram comparados pelo teste Qui quadrado a $95 \%$ de probabilidade.

\section{Resultados}

Com base nas informações do inventário $100 \%$ e romaneio das toras, a tabela 2 apresenta as estatísticas descritivas para as variáveis de DAP, $\mathrm{He} \mathrm{V}$ dos indivíduos de Lecythis lurida.

Tabela 2. Estatística descritiva para Lecythis lurida na área de manejo florestal da Coomflona, Floresta Nacional do Tapajós.

\begin{tabular}{ccccccc}
\hline & & $\mathrm{Y}$ & $\mathrm{s}$ & $\mathrm{CV} \%$ & Mínimo & Máximo \\
\hline \multirow{2}{*}{$\begin{array}{c}\text { Lecythis } \\
\text { lurida }\end{array}$} & $\mathrm{DAP}(\mathrm{cm})$ & 80,53 & 13,11 & 16,28 & 55,70 & 130,51 \\
& $\mathrm{H}(\mathrm{m})$ & 19,46 & 4,27 & 21,92 & 12,00 & 35,78
\end{tabular}


$\begin{array}{llllll}\mathrm{V}\left(\mathrm{m}^{3}\right) & 5,83 & 2,44 & 41,88 & 1,34 & 15,45\end{array}$

Em que: $\mathrm{Y}=$ média da variável de interesse; $\mathrm{s}=$ desvio padrão; $\mathrm{CV} \%$ = coeficiente de variação em porcentagem.

\section{Ajuste dos modelos volumétricos}

Após o ajuste dos modelos volumétricos, apenas Spurr variável combinada, Husch e Spurr e Schumacher Hall na forma logaritmizada apresentaram significância de todos os parâmetros, segundo o teste t a $95 \%$ de probabilidade (Tabela 3 ). Dentre estes, os modelos que apresentaram os maiores valores de $\mathrm{R}^{2}$ ajustado e menor valor de erro padrão de estimativa foram os modelos de dupla entrada Schumacher Hall e Spurr - ambos na forma logarítmica.

Tabela 3. Parâmetros dos ajustes e estatísticas de precisão para estimativas volumétricas de Lecythis lurida na área de manejo florestal da COOMFLONA, Floresta Nacional do Tapajós.

\begin{tabular}{|c|c|c|c|c|}
\hline \multirow{3}{*}{$\begin{array}{l}\text { Modelo } \\
\text { Spurr } \\
\text { variável } \\
\text { combinada }\end{array}$} & \multicolumn{2}{|c|}{ Coeficientes } & ajustado \% & Sy.x\% \\
\hline & $\beta 0$ & $1,261986^{*}$ & \multirow[b]{2}{*}{72,51} & \multirow[b]{2}{*}{23,24} \\
\hline & $\beta 1$ & $0,000035^{*}$ & & \\
\hline \multirow{2}{*}{$\begin{array}{l}\text { Spurr } \\
\text { logaritmizado }\end{array}$} & $\beta 0$ & $-8,164708^{*}$ & \multirow{2}{*}{75,12} & \multirow{2}{*}{23,15} \\
\hline & $\beta 1$ & $0,842790^{*}$ & & \\
\hline \multirow{2}{*}{ Husch } & $\beta 0$ & $-7,337934^{*}$ & \multirow{2}{*}{62,59} & \multirow{2}{*}{26,93} \\
\hline & $\beta 1$ & $2,065465^{*}$ & & \\
\hline \multirow{3}{*}{$\begin{array}{l}\text { Hohenald } \\
\text { Krenn }\end{array}$} & $\beta 0$ & $-3,088425^{\mathrm{ns}}$ & \multirow{3}{*}{63,75} & \multirow{3}{*}{26,68} \\
\hline & $\beta 1$ & $0,074088^{\mathrm{ns}}$ & & \\
\hline & $\beta 2$ & $0,000464^{\mathrm{ns}}$ & & \\
\hline Schumacher & $\beta 0$ & $-9,423173^{*}$ & \multirow{3}{*}{75,56} & \multirow{3}{*}{22,88} \\
\hline Hall & $\beta 1$ & $2,066793^{*}$ & & \\
\hline logaritmizado & $\beta 2$ & $0,696591^{*}$ & & \\
\hline \multirow{4}{*}{ Stoate } & $\beta 0$ & $-0,341864^{n s}$ & \multirow{4}{*}{73,12} & \multirow{4}{*}{22,98} \\
\hline & $\beta 1$ & $0,000355^{*}$ & & \\
\hline & $\beta 2$ & $0,000021^{*}$ & & \\
\hline & $\beta 3$ & $0,058364^{\mathrm{ns}}$ & & \\
\hline \multirow{5}{*}{$\begin{array}{l}\text { Meyer } \\
\text { modificado }\end{array}$} & $\beta 0$ & $-1,924541^{\mathrm{ns}}$ & \multirow{5}{*}{73,17} & \multirow{5}{*}{22,96} \\
\hline & $\beta 1$ & $0,043834^{\mathrm{ns}}$ & & \\
\hline & $\beta 2$ & $0,000070^{\mathrm{ns}}$ & & \\
\hline & $\beta 3$ & $0,000927^{\mathrm{ns}}$ & & \\
\hline & $\beta 4$ & $0,000018^{\mathrm{ns}}$ & & \\
\hline \multirow{6}{*}{ Meyer } & $\beta 0$ & $1,620725^{\mathrm{ns}}$ & \multirow{6}{*}{73,08} & \multirow{6}{*}{22,99} \\
\hline & $\beta 1$ & $-0,037432^{n s}$ & & \\
\hline & $\beta 2$ & $0,000521^{\mathrm{ns}}$ & & \\
\hline & $\beta 3$ & $0,004978^{\mathrm{ns}}$ & & \\
\hline & $\beta 4$ & $-0,000004^{\mathrm{ns}}$ & & \\
\hline & $\beta 5$ & $-0,177854^{\mathrm{ns}}$ & & \\
\hline
\end{tabular}

$\overline{\text { Em que }=\mathrm{NA}=\text { não se aplica; } \beta 0, \beta 1, \beta 2, \beta 3, \beta 4, \beta 5=\text { coeficientes }}$ da regressão; ns = coeficiente não significativo, de acordo com o teste $\mathrm{t}$ a $95 \%$ de probabilidade; $*$ = coeficiente significativo, de acordo com o teste $\mathrm{t}$ a $95 \%$ de probabilidade; Sy.x\% = erro padrão da estimativa.

No que diz respeito a multicolinearidade dos dados, entre os modelos com mais de dois coeficientes, o modelo de Schumacher Hall logarítmizado apresentou os menores valores de VIF, sendo valores de 1,07 para ambas variáveis independentes (Tabela 4). Já os modelos que apresentaram coeficientes não significativos, foi possível observar elevados valores de VIF, o que comprova que a multicolinearidade dos dados afeta a significância dos coeficientes.
Tabela 4. Valor de inflação da variância para modelos ajustados com mais de dois coeficientes.

\begin{tabular}{lcccccc}
\hline \multirow{2}{*}{ Modelo } & \multicolumn{6}{c}{ VIF } \\
\cline { 2 - 7 } & $\beta 0$ & $\beta 1$ & $\beta 2$ & $\beta 3$ & $\beta 4$ & $\beta 5$ \\
\hline Schumacher & & & & & & \\
$\begin{array}{l}\text { Hall } \\
\text { logaritmizado }\end{array}$ & - & 1,07 & 1,07 & & & \\
$\begin{array}{l}\text { Stoate } \\
\text { Meyer }\end{array}$ & - & 26,36 & 40,33 & 8,60 & & \\
modificado & - & 117,48 & 211,54 & 68,87 & 154,94 & \\
Meyer & - & 2420,80 & 2530,62 & 7423,84 & 7423,84 & 7423,84 \\
\hline
\end{tabular}

A distribuição gráfica dos resíduos mostrou que os modelos de Schumacher Hall (SH ln) e Spurr (SP ln), ambos na forma logarítmica, apresentaram tendências semelhantes. Já o modelo de Spurr variável combinada (SP var. comb) apresentou-se bem distribuído em torno da linha regressora (figura 2). 

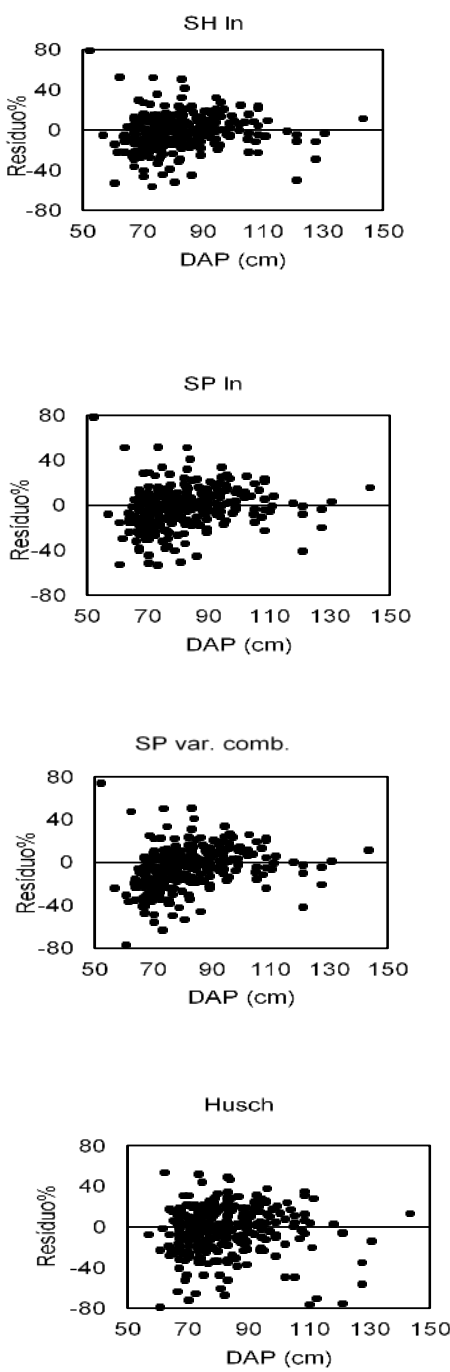

SH In

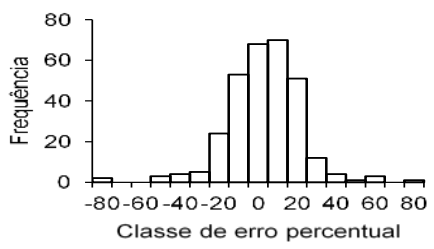

SP In

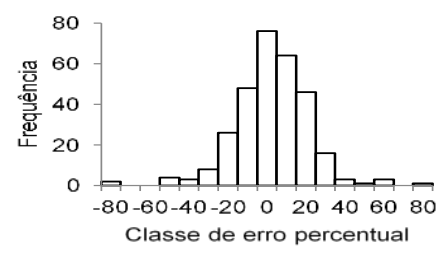

SP var. comb.

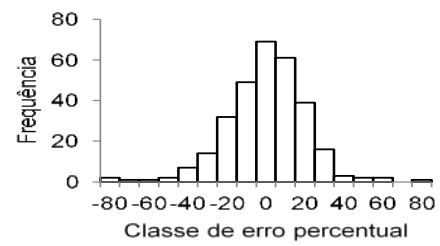

Husch

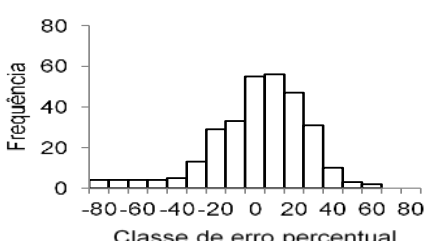

$\mathrm{SH} \ln$

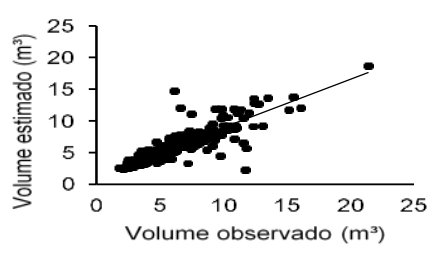

SP In

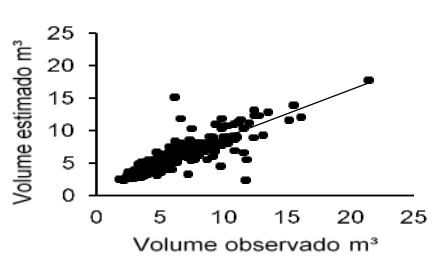

SP var. comb.

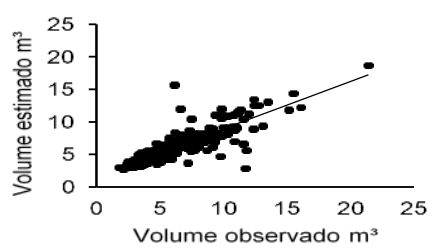

Husch

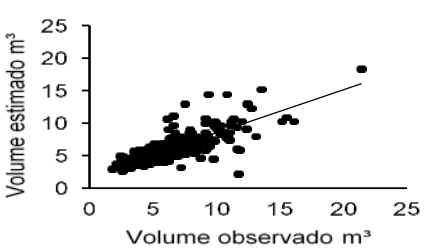

Figura 2. Distribuição gráfica de resíduos para a Lecythis lurida com os melhores modelos ajustados, na Floresta Nacional do Tapajós.

Os modelos de Spurr variável combinada, Spurr e Schumacher logaritmizados passaram pela padronização de resíduos, onde foi possível detectar que os modelos de Schumacher e Spurr variável combinada apresentaram 15 indivíduos considerados outliers e Spurr logaritmizado apresentou 16 outliers.

Após a padronização de resíduos, é possível perceber a melhora referente aos parâmetros da regressão para ambos modelos, sendo que o modelo de Schumacher passou a apresentar $\mathrm{R}^{2}$ ajustado de $87,64 \%$ e erro padrão de $14,64 \%$ e Spurr variável combinada apresentou $\mathrm{R}^{2}$ ajustado de $88,23 \%$ e erro padrão de $12,10 \%$, conforme mostra a tabela 5 .

Tabela 5. Parâmetros dos ajustes e estatísticas de precisão para os melhores modelos volumétricos após a padronização de resíduos.

\begin{tabular}{|c|c|c|c|c|c|c|}
\hline Modelo & Coeficientes & & & Q2 $^{2}$ austado $\%$ & $\mathrm{Sy} \times$ & VIF \\
\hline Número & $\beta 0$ & $\beta 1$ & $\beta 2$ & $\mathrm{~N}^{-}$- ajustauo $\%$ & & \\
\hline 1 & $0,74687^{*}$ & $0,00004 *$ & & 88,23 & 12,10 & Não se aplica \\
\hline 2 & $-8,81678^{*}$ & $0,89692 *$ & & 85,92 & 14,45 & Não se aplica \\
\hline 5 & $-9,21711 *$ & $2,00385^{*}$ & $0,72087 *$ & 87,64 & 14,64 & 1,051 \\
\hline
\end{tabular}

Em que: 1- Spurr variável combinada; 2 - Spurr logaritmizado; 3 - Schumacher Hall logaritmizado; $\beta 0, \beta 1, \beta 2, \beta 3=$ coeficientes da regressão; Sy. $\mathrm{x} \%$ = erro padrão da estimativa em porcentagem; $\mathrm{VIF}$ = fator de inflação da variância; * = coeficiente significativo, de acordo com o teste $\mathrm{ta}$ 95\% de probabilidade.

\section{Validação da melhor equação}

Ao aplicar a equação de Spurr variável combinada e comparar os volumes observados com os volumes estimados, demonstrou não haver diferença significativa entre os volumes com $\mathrm{p}$ valor $=0,4406$, a um nível de $95 \%$ de probabilidade, consequentemente validando o uso da equação para a área de estudo.

\section{Discussão}

Os modelos de dupla entrada foram estatisticamente superiores aos modelos de simples entrada. Isto ocorre, devido modelos de simples entrada assumirem que árvores de mesmo diâmetro possuem a mesma altura, o que não é verdadeiro para florestas heterogêneas (Scolforo 2005; Rolim et al. 2006; Soares et al. 2011b; Barreto et al. 2014). Além 
disso, modelos que apresentam duas variáveis independentes são apontados como mais eficientes, visto que em razão de suas propriedades estatísticas, esses modelos resultam quase sempre em estimativas mais precisas e não tendenciosas (Guimarães e Leite, 1996; Campos e Leite, 2009).

A multicolinearidade é uma complicação nos ajustes que ocorre, devido as variáveis independentes se apresentarem altamente correlacionadas (Ferreira 2009). Ocorre quando os modelos ajustados apresentam muitas variáveis correlacionadas e coeficientes não significativos na regressão, é indício de que ocorre multicolinearidade nos dados (Batista et al. 2004; Valente et al. 2011). Conforme Ferreira (2009), a multicolinearidade presente em alguns modelos, pode tornar os coeficientes da regressão imprecisos e consequentemente, as estimativas obtidas por esses coeficientes podem oscilar de um subconjunto de dados para o outro; por isso, aconselha-se a não utilização de modelos que apresentam multicolinearidade.

Apesar das análises dos parâmetros de qualidade e precisão dos ajustes fornecerem importantes requisitos para a escolha do melhor modelo, estas de forma isolada não garantem uma conclusão satisfatória sobre o desempenho deles, sendo necessário realizar a análise da distribuição gráfica dos resíduos (Miranda et al. 2014).

A distribuição de resíduos em torno da linha regressora dos gráficos para os modelos, apresentaram resultados com tendências semelhantes, porém ao analisar os outros critérios de seleção de melhor modelo, o modelo de Spurr variável combinada foi o mais adequado para estimar a volumetria da espécie na área. Modelos mais simples necessitam de um menor esforço computacional, são mais gerais e não sofrem com multicolinearidade. A grande difusão do modelo de Spurr na forma de variável combinada deve ser atribuída mais à facilidade de ajustamento, pois, com frequência, volumes de árvores menores são estimados com imprecisão (Couto e Bastos, 1987; Campos e Leite, 2009).

A escolha de modelos de dupla entrada como os mais precisos corrobora com resultados encontrados por outros autores na região. Para Thaines et al. (2010), o modelo de Schumacher Hall logarítmizado foi o modelo mais preciso para estimar o volume de madeira para a região da bacia do rio Ituxi. Barros e Silva Junior (2009), ao estudarem uma floresta localizada no município de Anapu, verificaram que o modelo de Spurr foi o mais adequado para estimar a volumetria dos indivíduos na área.

Tonini e Borges (2015), modelando o volume de árvores em Floresta Ombrófila Densa no sul de Roraima, observaram superioridade para os modelos de dupla entrada que empregaram a altura comercial como variável independente. Em um empreendimento florestal comunitário no Projeto de Desenvolvimento Sustentável Virola-jatobá no município de Anapu, Pará, Barreto et al. (2014), verificaram que o modelo proposto por Schumacher Hall se mostrou mais preciso para estimar o volume das espécies de interesse comercial, mostrando que a introdução da variável altura melhorou de forma significativa a precisão da equação ajustada.

Silva et al. (1984) ao realizarem ajuste de equação de volume para a espécie no $\mathrm{km} 67$ da FNT, identificaram que o modelo que mais se adequou para estimar o volume da Jarana foi Schumacher Hall. Já Moura (1994) também trabalhando com Jarana na FNT verificou o modelo de Prodan como mais preciso para estimar a volumetria da espécie, não descartando a utilização do modelo de Schumacher Hall e Meyer. SilvaRibeiro et al. (2014) ajustando modelos para Lecythis lurida na Floresta Nacional do Tapajós identificaram que os melhores modelos para estimar a volumetria da espécie foram Schumacher Hall e Spurr ambos logaritmizados.

\section{Conclusões}

O modelo de Spurr variável combinada, apresentou-se como o mais eficiente na estimativa volumétrica para Lecythis lurida na Floresta Nacional do Tapajós.

A equação $\mathrm{Vc} / \mathrm{c}=0,74687+0,00004 * \mathrm{DAP}^{2} *$ Hc é válida para estimar o volume de Lecythis lurida na área de estudo.

\section{Referências}

Andrade DF, Gama JRV, Melo LO, Ruschel AR (2015). Inventário florestal de grandes áreas na Floresta Nacional do Tapajós, Pará, Amazônia, Brasil. Biota Amazônia, Macapá, 5(1): 109-115.

Azevedo TL, Mello AA, Ferreira RA, Sanquetta CR, Nakajima NY (2011). Equações hipsométricas e volumétricas para um povoamento de Eucalyptus sp. localizado na FLONA do Ibura, Sergipe. Revista Brasileira de Ciências Agrárias, 6(1): 105-112.

Barreto WF, Leão FM, Menezes MC, Souza DV (2014). Equação de volume para apoio ao manejo comunitário de empreendimento florestal em Anapu, Pará.

Pesquisa Florestal Brasileira, Colombo, 34: 1-9.

Barros PLC, Silva-Júnior AT (2009). Equação de volume para árvores de uma floresta tropical densa no município de Anapu, oeste do estado do Pará, Amazônia oriental. Revista de Ciências Agrárias, 51 : $115-126$.

Batista JLF, Marquesini M, Viana VM. Equações de volume para árvores de caxeta (Tabebuia cassinoides) no Estado de São Paulo e sul do Estado do Rio de Janeiro. Scientia Forestalis, 65: 162-175.

BRASIL (2000). Ministério do Meio Ambiente. Decretolei $\mathrm{n}^{\circ}$ 9.985, de 18 de julho de 2000. Regulamenta o art. 225, § $1^{\circ}$, incisos I, II, III e VII da Constituição Federal, institui o Sistema Nacional de Unidades de Conservação da Natureza. Diário Oficial da República Federativa do Brasil, Brasília, DF, 18 jul. 2000. Seção 1, p. 1.

BRASIL (2009). Resolução Conama n ${ }^{\circ} \mathbf{4 0 6}$, de 2 de fevereiro de 2009. Estabelece parâmetros técnicos a serem adotados na elaboração, apresentação, avaliação técnica e execução de Plano de Manejo Florestal Sustentável-PMFS com fins madeireiros, para florestas nativas e suas formas de sucessão no bioma Amazônia. Ministério de Meio Ambiente, Brasília, DF, 4 p.

Cabacinha CD, Scolforo JRS, Thiersch CR, Sales NLP, Carvalho LR (2013). Uma nova abordagem para o método geométrico usando o índice da parábola. Revista Ciência Florestal, Santa Maria, 23(1): 261271.

Campos JCC, Leite HG (2009). Mensuração florestal: perguntas e respostas. 3 ed. Viçosa: Editora UFV, $542 \mathrm{p}$.

Couto HTZ, Bastos NLM (1987). Modelos de equações de volume e relação hipsométricas para plantações de Eucalyptus no Estado de São Paulo. IPEF, 37: 33-44.

Cysneiros VC, Pelissari AL, Machado SA, Figueiredo Filho A, Souza L (2017). Modelos genéricos e específicos para estimativa do volume comercial em 
uma floresta sob concessão na Amazônia. Scientia Forestalis, 45(45)

Equipe Estatcamp. Software Action. EstatcampConsultoria em estatística e qualidade, São Carlos SP, Brasil, 2014. Disponível em: http://www.portalaction.combr/. Acesso em: 19/09/2017.

Ferreira MZ (2009). Modelagem da influência de variáveis ambientais no crescimento e na produção de Eucalyptus sp., 112f. Tese. Universidade Federal de Lavras, Lavras.

Gomes KMA, Silva Ribeiro RB, Gama JRV, Andrade DFC (2018). Eficiência na estimativa volumétrica de madeira na Floresta Nacional do Tapajós. 6(2): 170176.

Guimarães DP, Leite HG (1996). Influência do número de árvores na determinação de equação volumétrica para Eucalyptus grandis. Scientia Forestalis, 50: 37-42.

IBGE - Instituto Brasileiro de Geografia e Estatística (2012). Manual técnico da vegetação brasileira. $2^{\mathrm{a}}$ ed. Revisada e ampliada, $271 \mathrm{p}$

IBAMA - Instituto Brasileiro do Meio Ambiente e dos Recursos Naturais Renováveis (2004). Floresta Nacional do Tapajós - Plano de Manejo. Brasília, 580p.

Leite FSL (2009). Estimativa do volume de madeira a partir do diâmetro da cepa em uma área explorada de floresta amazônica de terra firme. $74 \mathrm{p}$. Dissertação. Universidade de Brasília, Brasília.

Miranda LC, Paro BAV, Costa GR (2014). Estimativa do volume em árvores de Hymenaea coubaril L. e Trattinnickia burserifolia Mart. no norte de Mato Grosso. Nativa, Sinop, 2(4): 219-223.

Moura JB (1994). Estudo da forma do fuste e comparação de métodos de estimativa volumétrica de espécies florestais da Amazônia brasileira. 114p. Dissertação. Universidade Federal do Paraná, Curitiba.

Oliveira RCA, Rode R., Gama JRV, Almeida EC (2017). Equações volumétricas para Couratari stellata A.C Smith (Tauarí) na Floresta Nacional do Tapajós. Nativa, Sinop, 5(2): 138-144.

Pinho GSC, Fiedler NC, Guimarães PP, Silva GF, Santos J (2009). Análise de custos e rendimentos de diferentes métodos de corte de cipós para produção de madeira na floresta nacional do Tapajós. Acta Amazonica, 39: 555-560.

Rolim SG, Couto HTZ, Jesus RM, França JT (2006). Modelos volumétricos para a Floresta Nacional do Tapirapé-Aquirí, Serra dos Carajás (PA). Acta Amazonica, 36(1): 107 - 114.

Scolforo JRS 2005). Biometria florestal: parte I: modelos de regressão linear e não linear: parte II: modelos para relação hipsométricas, volume, afilamento e peso da matéria seca. Lavras: UFLA/FAEPE. $352 \mathrm{p}$.

SEMAS - Secretaria Estadual do Meio Ambiente e Sustentabilidade (2015). Instrução normativa $\mathbf{n}^{0} 5$ de 10/09/2015. Dispõe sobre procedimentos técnicos para elaboração, apresentação, execução e avaliação técnica de Plano de Manejo Florestal
Sustentável - PMFS nas florestas nativas exploradas ou não e suas formas de sucessão no Estado do Pará, e dá outras providências. Belém.

Silva JNM, Carvalho JOP, Lopes JCA, Carvalho MSP (1984). Equações de volume para a floresta nacional do Tapajós. Boletim de Pesquisa Florestal, Colombo, 8: 50-63.

Silva-Ribeiro RB, Gama JRV, Melo LO (2014). Seccionamento para cubagem e escolha de equações de volume para a Floresta Nacional do Tapajós. Cerne, 20: 605-612.

Soares CPB, Neto FP, Souza AL (2011 $\left.{ }^{\mathrm{a}}\right)$. Dendrometria e inventário florestal. Viçosa. Ed. UFV, 272p.

Soares CPB, Martins FB, Leite Junior HU, Silva GF, Figueiredo LTM (2011b). Equações hipsométricas, volumétricas e de Taper para onze espécies nativas. Revista Árvore, Viçosa, MG, 35(5): 1039 - 1051.

Taffarel M, Carvalho JOP, Melo LO, Silva MG, Gomes JM, Ferreira JER (2014). Efeito da silvicultura pós colheita na população de Lecythis lurida (Miers) Mori em uma floresta de terra firme na Amazônia brasileira. Ciência Florestal, Santa Maria, 24:887-896.

Thaines F, Braz EM, Mattos PP, Thaines AAR (2010). Equações para estimativa de volume de madeira para a região da bacia do Rio Ituxi, Lábrea, AM. Pesquisa Florestal Brasileira, Colombo, 30: 283-289, 2010.

Thomas C, Andrade CM, Scheneider PR, Finger CAG 2006). Comparação de equações volumétricas ajustadas com dados de cubagem e análise de tronco. Ciência Florestal, Santa Maria, 16: 319-327.

Tonini H, Borges RA (2015). Equação de volume para espécies comerciais em Floresta Ombrófila Densa no Sul de Roraima. Pesquisa Florestal Brasileira, 35: 111-117.

Trevisor TT 2011). Anatomia comparada do lenho de 64 espécies arbóreas de ocorrência natural na floresta tropical amazônica no estado do Pará. 217f. Dissertação. Universidade de São Paulo, Piracicaba.

Valente MDR, Queiroz WT, Pinheiro JG, Monteiro LAS (2011). Modelo de predição para o volume total de Quaruba (Vochysia imundata ducke) via análise de fatores e regressão. Revista Árvore, Viçosa - MG, 35: 307-317. 